\title{
Properties of Ultra Fast Deposited Diamond-Like Hydrogenated Carbon Films
}

\author{
J.L. Sullivan*, S.O. Saied And T. Zaharia \\ SEAS, Aston University, Birmingham, B4 7ET, United Kingdom
}

\begin{abstract}
Hydrogenated amorphous carbon films with diamond like structures have been formed on different substrates at very low energies and temperatures by a plasma enhanced chemical vapor deposition process employing acetylene as the precursor gas. The plasma source was of a cascaded arc type with Ar as carrier gas. The films were grown at very high deposition rates. Deposition on $\mathrm{Si}$, glass and plastic substrates has been studied and the films characterized in terms of $s p^{3}$ content, roughness, hardness, adhesion and optical properties. Deposition rates up to $20 \mathrm{~nm} / \mathrm{s}$ have been achieved at substrate temperatures below $100{ }^{\circ} \mathrm{C}$. The typical $s p^{3}$ content of $60-75 \%$ in the films was determined by X-ray generated Auger electron spectroscopy. Hardness, reduced modulus and adhesion were measured using a MicroMaterials Nano Test Indenter/Scratch tester. Hardness was found to vary from 4 to $13 \mathrm{GPa}$ depending on deposition conditions. Adhesion was significantly influenced by the substrate temperature and in situ DC cleaning. Hydrogen content in the film was measured by a combination of the Fourier transform infrared and Rutherford backscattering techniques. Advantages of these films are: low ion energy and deposition temperature, very high deposition rates, low capital cost of the equipment and the possibility of film properties being tailored according to the desired application.
\end{abstract}

PACS: 81.15.- z, 52.77.-j

\section{Introduction}

In many engineering and medical applications, materials which are mechanically most suited for the production of application specific devices, in many circumstances, will not fulfil the criteria of, for example, low friction and wear, environmental compatibility or bio-compatibility. Hence, the requirement for surface coatings. Interest in deposited amorphous carbon thin films has been growing over the last two decades due to their unique properties of high hardness, chemical inertness, low friction and low wear. Hydrogenated films proved to be particularly useful, since although the ultimate density and hardness may be lower than for unhydrogenated films they tend to be more mechanically compliant and less liable to delaminative failure. Hydrogen also enhances the deposition rates and transforms $s p^{2}$ bonds into $s p^{3}$ bonds in the process of hydrogenation [1]. Thus hydrogenated amorphous carbon films (a-C:H) are an ideal candidate for the coating of medical devices [2-7]. In addition, the physical and chemical properties of a-C:H can be tailored to give optimum short term compatibility with their specific environment by alteration of deposition conditions and doping.

A major advantage of the a-C:H films discussed here is that they can be produced at low ion energy of less than $0.5 \mathrm{eV}$, very high deposition rates and low surface temperature, thus minimising damage to temperature sensi-

* corresponding author; e-mail: j.l.sullivan@aston.ac.uk tive substrate materials, such as plastics. According to the existing deposition models, high $s p^{3}$ content material should not be formed at these low energies. Sub-plantation models, proposed by Robertson [8] and Lifshitz et al. [9], assumes that subsurface implantation of energetic species generates an increase in density, which leads to an increase in $s p^{3}$ bonding. In his review of the subject, Robertson [8] suggests that the best quality unhydrogenated material is produced at an optimum energy of around $100 \mathrm{eV}$ per incident carbon atom. The model assumes that at energies lower than $30-40 \mathrm{eV}$ penetration of the surface does not occur and therefore diamond-like carbon material (high $s p^{3}$ content) should not be formed.

An alternative thermal spike model, proposed by Hofsass [10] assumes that all excess energy generated by ion impact is converted to thermal energy which then diffuses outwards, favouring $s p^{3}$ bonding during atomic rearrangements. According to this model, the required energy for $s p^{3}$ bond formation falls in the range $50-2000 \mathrm{eV}$.

McKenzie et al. [11] suggested that the compressive stress generated during film growth is the cause of the high $s p^{3}$ bond content in the films, where the optimum energy for formation lies in the interval 15 to $70 \mathrm{eV}$. In the model proposed by Koponen et al. [12] the formation of diamond-like bonding is attributed to transient high pressure created by the energetic ion impact. The relaxation to energetically favoured graphitic state is assumed to proceed through a succession of metastable states.

Even the more recent molecular simulations by Marks [13] show that a threshold energy of around $6 \mathrm{eV}$ is required for the formation of $s p^{3} \sigma$ bonding in these 
films. These simulations are based on a model of surface insertion in which energetic burial leads to the simultaneous processes of $s p^{3}$ promotion, densification, stress generation and surface growth.

All the above models apply to the formation of unhydrogenated films. In the case of hydrogenated films, the film growth mechanism is more difficult to model, but it is assumed to resemble the unhydrogenated growth models, and the energies involved (per carbon atom) are assumed to be similar [8].

The one common factor connecting all of the models is that energies far greater than those generated in our experiments are necessary for the formation of $\sigma$ bonding in these amorphous carbon films. However, this approach only considers the physical interactions, whereas in fact chemical processes may be the driving factor at very low impact energies. Recent studies have shown that the growth of good quality hydrogenated carbon films at very low energies is a result of the presence of specific radicals $\left(\mathrm{C}_{3}, \mathrm{C}_{3} \mathrm{H}\right)$. The unsaturated bonds of these radicals are preserved by the absence of high energy bombardment. Thus films are produced with low defect density and relative high hardness $(12-15 \mathrm{GPa})$ due to the transformation of polymeric $s p^{3} \mathrm{C}-\mathrm{H}$ bonds into $s p^{3} \mathrm{C}-\mathrm{C}$ bonds that form an interconnecting $s p^{3}$ network [14].

In the work described here hydrogenated a-C:H films have been formed at ion energies of less than $1 \mathrm{eV}$, at high deposition rates and low substrate temperatures (less than $100^{\circ} \mathrm{C}$ ), using a cascaded arc plasma enhanced chemical vapor deposition (PECVD) process. The films were deposited on Si (100), glass and plastic (PET, PEEK) substrates using acetylene as precursor gas, injected in two different positions, at different acetylene to argon flow rates and at two different substrate temperatures. The films were analysed as a function of the plasma parameters in terms of $s p^{3}$ fraction, hardness, reduced modulus, hydrogen content and adhesion.

\section{Experimental setup}

The deposition cell is shown in Fig. 1. The plasma source is a cascaded arc employing Ar as the carrier gas. This creates an expanding thermal plasma (ETP) in the vacuum chamber [15-17]. When the precursor gas (acetylene) is injected via an injection ring the gas molecules are dissociated and ionised and this gives rise to a high flux of particles (neutrals and ions) directed towards the substrate. Typical ionisation in the plasma jet is between 2 to $10 \%$ of the total carrier gas volume, depending on the power injected in the cascaded source and the flow of the carrier gas [1]. The setup has the advantage of separating the functions of production, transport and deposition, which facilitates doping, adjusting type, quality and growth rates for the deposited films.

Pressures and flow rates during each experimental run were maintained in the vacuum chamber by a roots blower pump. The pumping speed was varied with a frequency inverter which resulted in operating pressures

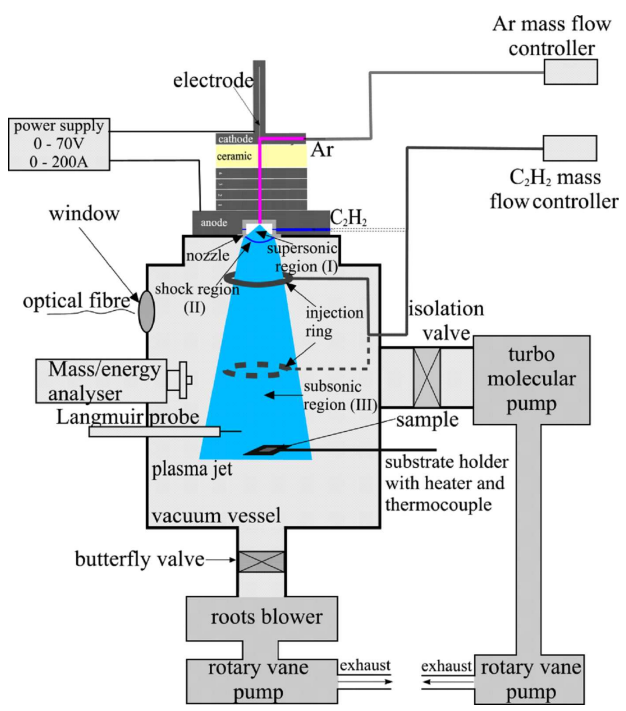

Fig. 1. The schematics of the experimental setup.

during deposition of $0.5-0.6$ Torr. The Si (100) and glass substrates (dimension $8 \times 8 \mathrm{~mm}^{2}$ ) were ultrasonically cleaned in distilled water, neutrocon detergent and isopropyl alcohol. They were then secured to a substrate holder with silver paste and inserted into the vacuum chamber. The sample holder was constructed of nickel plated copper, due to the incompatibility between acetylene and copper. All the substrates ( $\mathrm{Si}$, glass, PET, PEEK) were ion cleaned in situ, prior to the deposition process, with a DC plasma air glow discharge for 15-20 min. The vacuum chamber was pumped down to a base pressure of less than $5 \times 10^{-6}$ mbar using a turbomolecular pump. The injected power in the plasma was varied from 1.2 to $3 \mathrm{~kW}$. The separation between substrate and source could be varied up to a maximum of $90 \mathrm{~cm}$. Two different positions for the injection of the precursor gas could be used, i.e. via the injection ring $(\Phi=8 \mathrm{~cm})$ situated at $9 \mathrm{~cm}$ from the nozzle of the source or half way between source and substrate, at a distance of $37 \mathrm{~cm}$ away from the nozzle. At a later stage a new substrate holder was designed, capable to hold larger substrates (dimensions $50 \mathrm{~mm} \times 50 \mathrm{~mm}$ ). The holder was resting on the bottom of the vacuum chamber and an additional steel sheet was slid vertically from the top. The samples were secured with silver paste to this steel sheet, to maintain a good temperature control.

The deposition time was kept under $60 \mathrm{~s}$ for most of the samples, to maintain a small variation in sample temperature during the deposition process. This still allowed films with a thickness of 400-500 $\mathrm{nm}$ to be formed.

The plasma Ar jet was analysed using a Hiden Mass/ Energy analyser and a Hiden Langmuir electrical probe to monitor the species, concentrations and energy of the ions. Measurements during the deposition process were not possible, due to the very fast deposition rates, which effectively covered the entrance aperture of the energy analyser and the tip of the Langmuir probe in seconds. 
The topography and surface roughness of the films was mapped with a Thermo Microscopes M5 atomic force microscope. The instrument was used in contact mode and the scanned area varied from $10 \mu \mathrm{m} \times 10 \mu \mathrm{m}$ to $100 \mu \mathrm{m} \times 100 \mu \mathrm{m}$.

The thickness and refractive index of the films were measured by a Rudolph Research Auto EL III ellipsometer with a $632.8 \mathrm{~nm}$ wavelength laser. The films grown at very fast growth rates (more than $10 \mathrm{~nm} / \mathrm{s}$ ) are optically absorbent and ellipsometry is not a good technique for analysing these absorbent films. Therefore thickness was measured by masking a small area of the sample with a temperature resistant tape during deposition and then measuring the step formed using a profilometer.

$s p^{3}$ fractions were determined by X-ray Auger electron spectroscopy (XAES) technique [18-23] in a Thermo Fisher 250i spectrometer. The X-ray source was $\mathrm{Al} K_{\alpha}$ $(1486.6 \mathrm{eV})$. The $\mathrm{C} 1 s$ and $\mathrm{O} 1 s$ peaks were collected with a pass energy of $20 \mathrm{eV}$ at steps of $0.1 \mathrm{eV} /$ channel, the C $K L L$ spectra collected with a pass energy of $20 \mathrm{eV}$ in steps of $0.2 \mathrm{eV} /$ channel. The samples were stored in plastic boxes after deposition and then inserted in the X-ray photoemission spectroscopy (XPS) analysis chamber in batches of 6 . The analysed area was $2 \times 3 \mathrm{~mm}^{2}$ for each sample and the base pressure during the analysis was less than $5 \times 10^{-9}$ mbar.

The relative hydrogen content in the films was measured with a FTIR Bruker Vector 22 spectrometer. The software used to collect and analyse the spectra was Opus 4.0. First a virgin silicon sample was measured, to find a background level, then the absorption spectra were collected for all samples and the silicon signal subtracted. Care was taken when preparing the samples for analysis, i.e. all traces of silver paste were carefully removed from the back side of the silicon sample, because silver peaks interfere with the a-C:H peaks. The absolute hydrogen content was measured for few samples (with different relative hydrogen content) using the Rutherford backscattering (RBS) technique. The results were then compared and the relative values converted to absolute ones.

Hardness and reduced modulus was measured with a Micro Materials NanoTest 500 instrument using a Berkovich indenter. The indentation depth was kept to $10-20 \%$ of the film thickness to minimise the influence from the Si substrate [24-27]. The temperature in the cabinet was kept constant at 2 degrees above the room temperature. The samples were glued with superglue to a stub and removed by heating afterwards. The indenter was periodically calibrated and standard tests were performed daily. Scratch tests were performed on each sample with a Rockwell indenter. The scratch length was $500 \mu \mathrm{m}$ and the maximum force applied during the tests was typically $70-200 \mathrm{mN}$. To quantify the adhesion of the film to the substrate the critical loading divided by the film thickness was calculated and compared for each sample.

\section{Results and discussion}

\subsection{Plasma analysis}

The energy analyser indicated that the energy of the Ar ions in the region close to the sample holder was around $0.3 \mathrm{eV}$, with small variations related to the process pressure. The Langmuir probe current-voltage characteristic showed that the Ar ions density in the downstream region was $3.5 \times 10^{18} \mathrm{~m}^{-3}$, comparable to the values found by others $[1,28]$.

\subsection{AFM topography}

AFM mapping of the film's surface revealed that Ra values of the film surfaces generally followed the Ra values of the substrates. Figure 2 shows typical examples of films formed on different substrates.

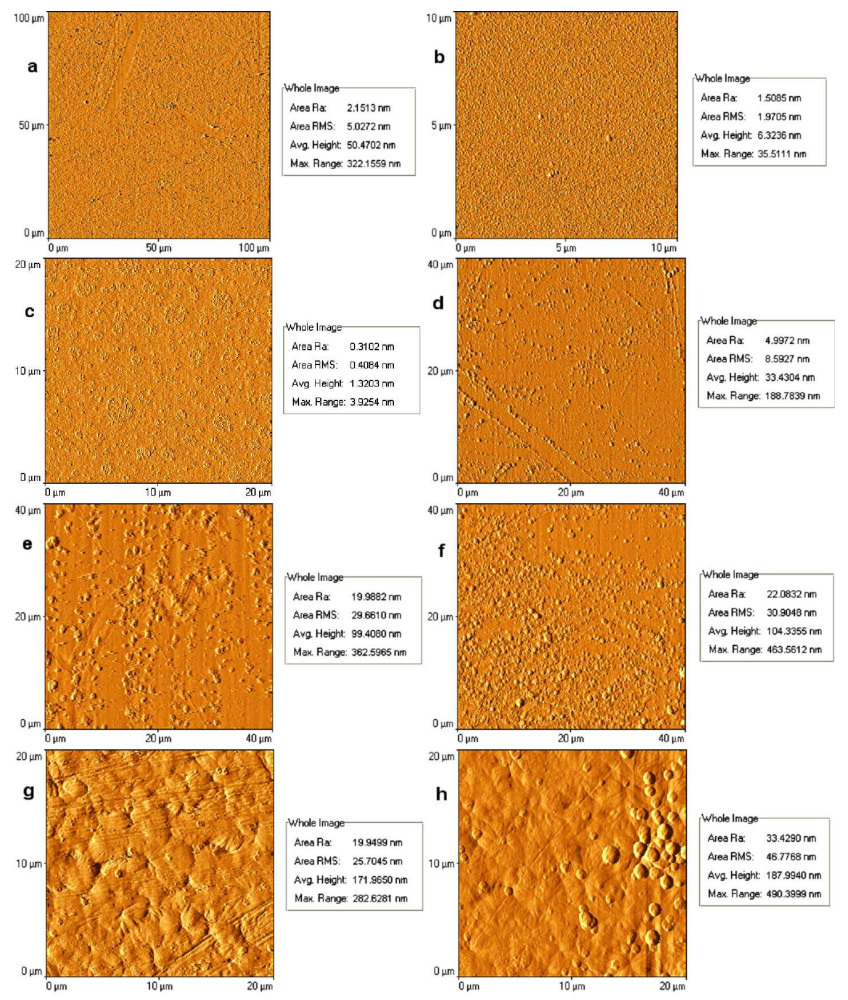

Fig. 2. AFM images of (a) virgin silicon substrate, (b) $\mathrm{a}-\mathrm{C}: \mathrm{H}$ film on silicon, (c) virgin glass substrate, (d) a-C:H film on glass, (e) virgin PET substrate, (f) a-C:H film on PET, (g) virgin PEEK substrate, and (h) a-C:H film on PEEK.

\subsection{Deposition rates and optical properties}

The growth rates increased with the relative flow of $\mathrm{C}_{2} \mathrm{H}_{2}$ to Ar. The deposition rates are higher when acetylene is injected with the ring close to the source $(9 \mathrm{~cm}$ from the nozzle) by a factor of at least 2 . This is because when the injection ring is in its lower position $(37 \mathrm{~cm}$ from nozzle) the plasma jet is attenuated by the ring itself. Deposition rates as a function of $\mathrm{C}_{2} \mathrm{H}_{2}$ :Ar flow are 


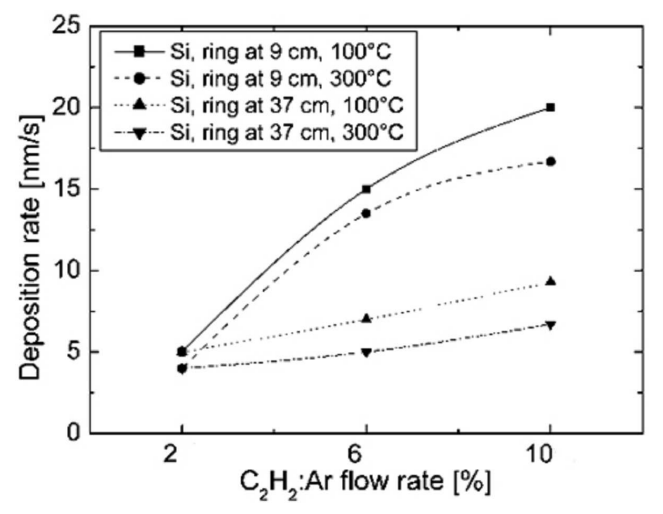

Fig. 3. Deposition rates against $\mathrm{C}_{2} \mathrm{H}_{2}$ : Ar flow rate at $0.5 \mathrm{Mbar}$ operating pressure for films grown on $\mathrm{Si}$ substrate at deposition temperatures of $<100^{\circ} \mathrm{C}$ and $300^{\circ} \mathrm{C}$ with injection ring at 9 and $37 \mathrm{~cm}$ from the nozzle of the source.

shown in Fig. 3. In the next phase of the project an injection ring with wider diameter will be used for the lower position.

The refractive index was measured with the ellipsometer only for the films deposited on $\mathrm{Si}$ at low $\mathrm{C}_{2} \mathrm{H}_{2}$ : $\mathrm{Ar}$ flows and gave values of 1.85-1.95. The films deposited at higher flow rates are denser and have a refractive index higher than 1.95 .

\section{4. $s p^{3}$ fraction (XAES)}

The derivative of the XAES spectra was obtained by a Savinsky-Golay method, after a smoothing with 27 points, 2 passes was performed. The difference between the mean of the positive and negative peaks in the differentiated XAES spectra $(D)$ was monitored for all films, examples of this are shown in Fig. 4. From the analysis of a Nixon industrial diamond and a pure graphitic sample two extreme values for $D$ of $14.3 \mathrm{eV}$ and $22.5 \mathrm{eV}$ were found, respectively, identical to those reported by Lascovich [19]. The $s p^{3}$ fractions were then deduced for all the a-C:H films assuming a linear dependence between the diamond and graphite values [19].

The $\mathrm{C} 1 s$ and $\mathrm{O} 1 s \mathrm{X}$-ray photoelectron spectra were also collected and smoothed with 9 points and 10 passes. A typical surface contamination of $7-10 \%$ atomic concentration of oxygen was found for all the a-C:H films analysed. The $\mathrm{C} 1 s$ peak was fitted assuming a difference of $0.7-0.8 \mathrm{eV}[20]$ between the deconvoluted $s p^{2}$ and $s p^{3}$ peaks. The values for $s p^{3} / s p^{2}$ ratio from the XPS C1s fit were found to be in good correlation with the ones found from the Auger first derivative spectra, even though the penetration depth is $7.5 \mathrm{~nm}$ in the case of XPS, compared to only $2.0-2.5 \mathrm{~nm}$ for the XAES method [29, 30].

As it can be seen from the graph in Fig. 5, all the films showed relatively high $s p^{3}$ fractions, decreasing from low $\mathrm{C}_{2} \mathrm{H}_{2}$ :Ar relative flows $(2 \%)$ to medium/high $\mathrm{C}_{2} \mathrm{H}_{2}$ : $\mathrm{Ar}$ relative flow $(6-10 \%)$.

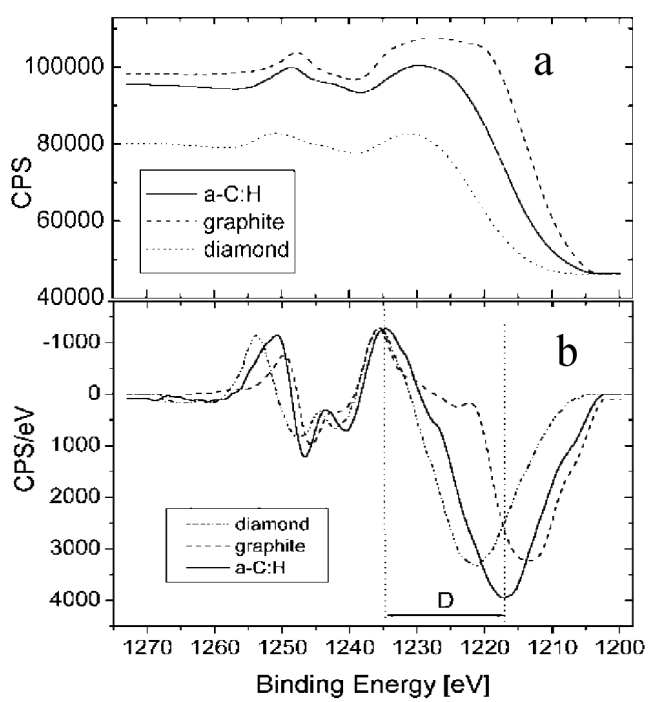

Fig. 4. Integral $N(E)$ XAES spectra of graphite, diamond and a hard a-C:H film (left image); derivative $\mathrm{d} N(E) / \mathrm{d} E$ XAES spectra of graphite, diamond and a hard a-C:H film (right image).

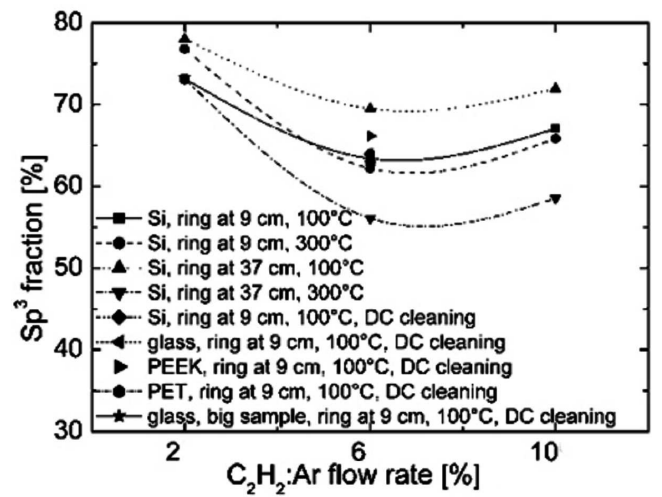

Fig. 5. $s p^{3}$ fraction for films grown on silicon from acetylene injected at two different ring positions ( 9 and $37 \mathrm{~cm}$ away from the nozzle) and at two different deposition temperatures as calculated by XAES.

\subsection{Hydrogen content}

The relative hydrogen content in the film was calculated assuming that the area of the absorption spectrum for each sample is directly proportional to the number of $\mathrm{C}-\mathrm{H}$ bonds and thus to the hydrogen content of the film [1]. The relative values were then transformed to absolute values], by comparing the results of two identical films, analysed with the Fourier transform infrared (FTIR) and RBS, respectively.

A clear trend can be observed from Fig. 6. The hydrogen content of the films decreased with the increase in the relative $\mathrm{C}_{2} \mathrm{H}_{2}$ :Ar flow from low values $(2 \%)$ to medium/ high values $(6-10 \%)$. This decrease is more abrupt for the films grown when acetylene is injected in the "ring up" position. 


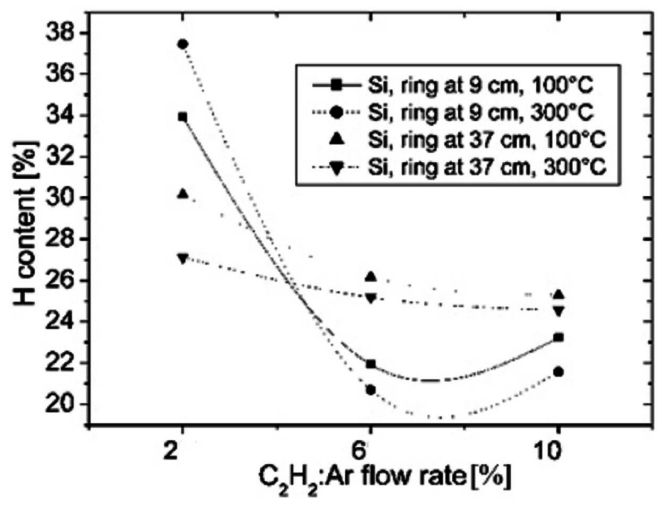

Fig. 6. Absolute hydrogen content for films grown at $100{ }^{\circ} \mathrm{C}$ and $300^{\circ} \mathrm{C}$ substrate temperature on silicon from acetylene injected via the ring situated at 9 or $37 \mathrm{~cm}$ from the plasma source.

\subsection{Hardness, reduced modulus and adhesion}

The software provided with the Nano Tester automatically analyses the loading-unloading curves and calculates the hardness and reduced modulus of the films at the desired indentation depth. The results are presented in Fig. 7 and Fig. 8.

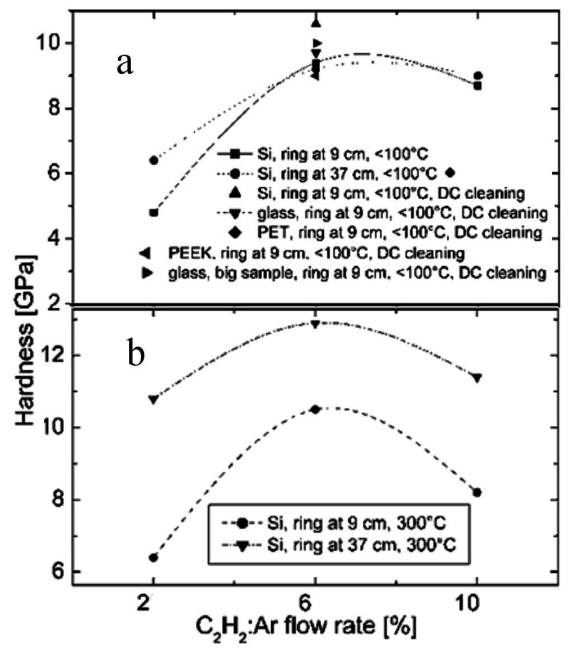

Fig. 7. Hardness against $\mathrm{C}_{2} \mathrm{H}_{2}$ relative flow for films grown from $\mathrm{C}_{2} \mathrm{H}_{2}$ injected via the ring (a) on different substrates under different conditions at temperatures $<100{ }^{\circ} \mathrm{C}$ and (b) on silicon at $300^{\circ} \mathrm{C}$ deposition temperature.

The analysis showed hardness and reduced modulus to increase to a maximum at a $\mathrm{C}_{2} \mathrm{H}_{2}$ to $\mathrm{Ar}$ ratio of about $6 \%$ in the first set of experiments performed on silicon substrates, at $100{ }^{\circ} \mathrm{C}$ and $300{ }^{\circ} \mathrm{C}$ substrate temperatures. In the second set of experiments, when the best conditions were applied to different substrates at low temperatures $\left(<100^{\circ} \mathrm{C}\right)$, the hardness remained at a value of around $10 \mathrm{GPa}$, except for the PES substrate. This was due to the very soft substrate which probably deformed

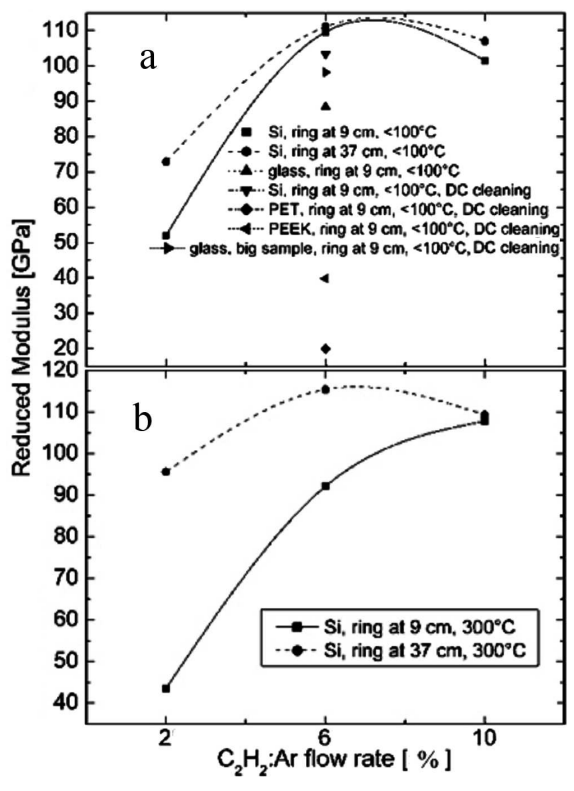

Fig. 8. Reduced modulus against $\mathrm{C}_{2} \mathrm{H}_{2}$ relative flow for films grown from $\mathrm{C}_{2} \mathrm{H}_{2}$ injected via the ring (a) on different substrates under different conditions at temperatures $<100{ }^{\circ} \mathrm{C}$ and (b) on silicon at $300^{\circ} \mathrm{C}$ deposition temperature.

before the film did. The films deposited at higher temperatures $\left(300^{\circ} \mathrm{C}\right)$ on $\mathrm{Si}$ showed an increased hardness, clearly observed in the experiments with acetylene injected through the ring situated at $37 \mathrm{~cm}$. The same trends were observed for the reduced modulus, the only significant difference being that the elastic properties of both films deposition on plastic (PES, PEEK) were influenced by the softer substrate.

Comparing the topography of the surface before and after each scratch allowed the identification of the critical loading. This value was then divided by the thickness of each film. The results are shown in Fig. 9. The adhesion was found to improve significantly (by a factor of $\approx 2$ ) when the in situ cleaning DC glow discharge was employed for films deposited at low temperatures $\left(<100^{\circ} \mathrm{C}\right)$. For the films deposited on plastics (PEEK, PES) a critical loading value could not be determined, the scratch pattern being completely different to the pattern observed on silicon or glass substrates.

The $s p^{3}$ fractions shown in Fig. 5 are higher than might be expected for this type of a-C:H film and this is due to the fact that XAES cannot differentiate between $\mathrm{C}-\mathrm{C}$ and $\mathrm{C}-\mathrm{H}$ bonds. It is assumed that at low $\mathrm{C}_{2} \mathrm{H}_{2}$ :Ar flow ratio $(2 \%)$ a large proportion of the $s p^{3}$ bonds come from polymeric $\mathrm{C}-\mathrm{H}$ bonds, due to high hydrogen incorporation in the film. As the $\mathrm{C}_{2} \mathrm{H}_{2}$ :Ar ratio increases $(6 \%)$, the total $s p^{3}$ fraction decreases due to a reduction of the hydrogen in the film. This leads to a decrease in the number of polymeric $\mathrm{C}-\mathrm{H} s p^{3}$ bonds, whereas the increase in hardness suggests that the number of $\mathrm{C}-\mathrm{C} s p^{3}$ bonds has increased. Around this relative flow a saturation point is 


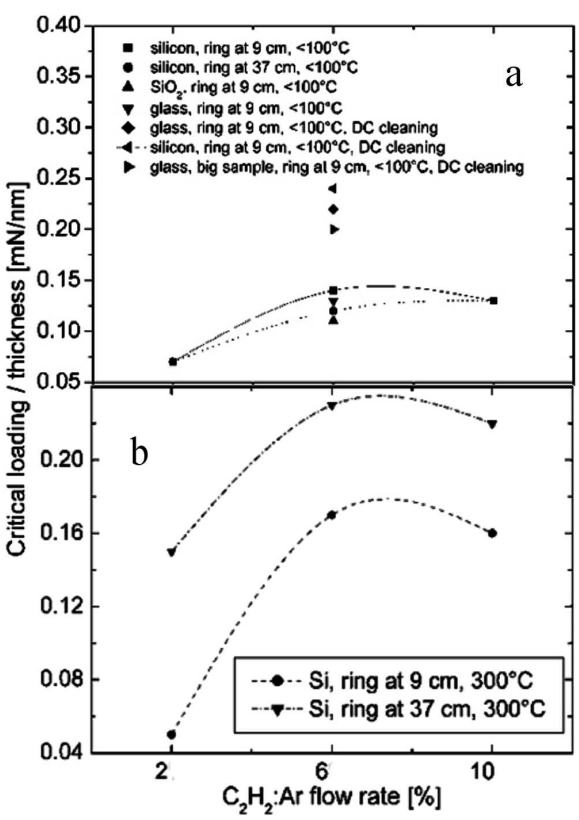

Fig. 9. Adhesion against $\mathrm{C}_{2} \mathrm{H}_{2}$ relative flow for films grown from $\mathrm{C}_{2} \mathrm{H}_{2}$ injected via the ring (a) on different substrates under different conditions at temperatures $<100{ }^{\circ} \mathrm{C}$ and (b) on silicon at $300^{\circ} \mathrm{C}$ deposition temperature.

reached and at even higher $\mathrm{C}_{2} \mathrm{H}_{2}$ :Ar flow ratio (10\%) the variations in the $s p^{3}$ fraction are small. This is in good agreement with the measurement performed by FTIR/ RBS, that showed a decrease in the hydrogen content in the films from low $\mathrm{C}_{2} \mathrm{H}_{2}$ :Ar flows $(2 \%)$ to medium/high $\mathrm{C}_{2} \mathrm{H}_{2}$ :Ar flows (6-10\%) (see Fig. 6), clearly visible when the injection of acetylene takes place closer to the nozzle of the source.

The hardness and modulus measurements show that the best films are formed at $\mathrm{C}_{2} \mathrm{H}_{2}$ :Ar relative flows higher than $6 \%$. There are small differences in the hardness of the films deposited with acetylene injected at different positions (ring at 9 and $37 \mathrm{~cm}$ respectively), more clearly observed at high deposition temperatures. This is due to differences in plasma chemistry at the substrate level and variations in the sticking coefficient of the different radicals onto the growing film with temperature. It is now believed that the main radicals that contribute to the film growth are $\mathrm{C}_{3}$ and $\mathrm{C}_{3} \mathrm{H}$ in the presence of atomic $\mathrm{H}$. The hardness of the films is determined by the $\mathrm{C}-\mathrm{C} s p^{3}$ bonding and the amount of $\mathrm{H}$ in the films. The reduced modulus was significantly lower for the films deposited on plastic substrates, which suggests that the substrate contribution is still important for these hard films on softer substrates.

\section{Conclusion}

Hydrogenated amorphous carbon films with diamond like properties have been deposited using acetylene as a precursor gas at very low energies and low deposition temperatures on different substrates. The structure and properties of the films formed on different substrates were similar, as revealed by the AFM examination; XAES derived $s p^{3}$ fractions and hardness measurements. Scratch tests showed similar results for the adhesion of films on deposited silicon and glass substrates. For the films deposited on the plastics PET and PEEK results were difficult to interpret quantitatively due to deformation of the substrates, but subjectively it was felt that good adhesion was also exhibited on these materials.

The growth rates for these films were very high, $\approx 20 \mathrm{~nm} / \mathrm{s}$ which is up to 100 times greater than other PECVD methods. The surface of the roughness of the films was found to be at least as good as the substrate on which they were deposited, of the order of $2 \mathrm{~nm} \mathrm{Ra}$ for Si. The refractive index, an indicator of film density, was high $(>1.95)$, the $s p^{3}$ fraction was $60-75 \%$ and the hardness between 9 and $11 \mathrm{GPa}$ at deposition temperatures below $100^{\circ} \mathrm{C}$. The reduced elastic modulus for these films was found to be in the range 90-110 GPa. The adhesion to substrates was found to be good at high deposition temperatures on $\mathrm{Si}$ and glass and to increase to similar levels to this for all materials by in situ cleaning prior to deposition at low deposition temperatures. The films were, however, found to have a practical thickness limit of $\approx 1.5 \mu \mathrm{m}$ above which delamination from the substrate occurred due, presumably, to internal compressive stresses.

All of the above properties are comparable with good quality films deposited by other PECVD methods. The advantages, however, are the low energy and temperature of deposition, ensuring that no damage is done to sensitive substrates; very high deposition rates and relatively low capital cost of the equipment required. Also the ease of adjustment of plasma parameters will allow film properties to be tailored according to the desired application (different hardness, hydrogen incorporation, doping).

\section{References}

[1] J.W.A.M. Gielen, Ph.D. Thesis, Technical University of Eindhoven, 1996.

[2] G. Dearnaley, J.H. Arps, Surf. Coat. Technol. 200, 2518 (2005).

[3] R. Hauert, Diamond Rel. Mater. 12, 583 (2003).

[4] P.D. Maguire, Diamond Rel. Mater. 14, 1277 (2005).

[5] V.-M. Tiainen, Diamond Rel. Mater. 10, 153 (2001).

[6] R.J. Narayan, Mater. Sci. Eng. C 25, 405 (2005).

[7] R. Hauert, Tribol. Int. 37, 991 (2004).

[8] J. Robertson, Mater. Sci. Eng. $R$ 37, 129 (2002).

[9] Y. Lifshitz, S.R. Kasi, J.W. Rabalais, W. Eckstein, Phys. Rev. B 41, 10468 (1990).

[10] H. Hofsass, H. Feldermann, R. Merk, M. Sebastian, C. Ronning, Appl. Phys. A 66, 153 (1998).

[11] D.R. McKenzie, D. Muller, B.A. Pailthorpe, Phys. Rev. Lett. 67, 773 (1991). 
[12] I. Koponen, M. Hakovirta, R. Lappalainen, J. Appl. Phys. 78, 5837 (1995).

[13] N.A. Marks, Diamond Rel. Mater. 14, 1223 (2005).

[14] J. Benedikt, Ph.D. Thesis, Technical University of Eindhoven, 2004.

[15] S. Mazouffre, Ph.D. Thesis, Technical University of Eindhoven, 2001.

[16] M.C.M. van de Sanden, J.M. de Regt, D.C. Schram, Plasma Sources Sci. Technol. 3, 501 (1994).

[17] R.F.G. Meulenbroeks, R.A.H. Engeln, M.N.A. Beurskens, Plasma Sources Sci. Technol. 5, 74 (1995).

[18] J.C. Lascovich, R. Giorgi, S. Scaglione, Appl. Surf. Sci. 47, 17 (1991).

[19] J.C. Lascovich, S. Scaglione, Appl. Surf. Sci. 78, 17 (1994).

[20] P. Merel, M. Tabal, M. Chaker, S. Moisa, J. Margot, Appl. Surf. Sci. 136, 105 (1998).

[21] P. Patsalas, M. Handrea, S. Logothetidis, M. Gioti, S. Kennou, W. Kautek, Diamond Relat. Mater. 10, 960 (2001).

[22] J. Filik, P.W. May, S.R.J. Pearce, R.K. Wild, K.R. Hallam, Diamond Relat. Mater. 12, 974 (2003).
[23] E.C. Samano, Appl. Surf. Sci. 202, 1 (2002).

[24] J.F. Lin, P.J. Wei, J.C. Pan, C.-F. Ai, Diamond Relat. Mater. 13, 42 (2004).

[25] H. Kim, D.H. Jung, B. Park, K.C. Yoo, J.J. Lee, J.H. Joo, Surf. Coat. Technol. 193, 255 (2005).

[26] P. Bruno, G. Cicala, A.M. Losacco, P. Decuzzi, Surf. Coat. Technol. 180-181, 259 (2004).

[27] J.P. Quinn, P. Lemoine, P. Maguire, J.A. McLaughlin, Diamond Relat. Mater. 3, 1385 (2004).

[28] M.F.A.M. van Hest, J.R. Haartsen, M.H.M. van Weert, D.C. Schram, M.C.M. van de Sanden, Plasma Sources Sci. Technol. 12, 539 (2003).

[29] M.P. Seah, W.A. Dench, Surf. Anal. 1, 2 (1979).

[30] J.F. Watts, J. Wolstenholme, An Introduction to Surface Analysis by XPS and AES, John Wiley and Sons, New Jersey 2003, p. 92. 